\title{
Las 3 etapas de las prácticas pre-profesionales como parte de la aplicación eficiente del conocimiento de estudiantes en unidades educativas
}

\section{The 3 stages of pre-professional practices as part of the efficient application of student knowledge in educational units}

\author{
Mg. Dorys Maribel Cumbe Coraizaca ${ }^{1}$ \\ dm.cumbe@uta.edu.ec \\ Mg. Laura Cristina Andaluz Espejo ${ }^{2}$ \\ Ic.andaluz@uta.edu.ec \\ Mg. Ana Jazmine Vera de la Torre ${ }^{3}$ \\ aj.vera@uta.edu.ec
}

Recibido: 1/09/2017, Aceptado: 1/11/2017

\begin{abstract}
RESUMEN
En el mundo actual, la mayor parte de instituciones solicitan docentes con experiencia en enseñanza y son justamente; las prácticas pre profesionales, las que les brindan a los estudiantes la experiencia requerida, porque se involucran en el mundo real donde al culminar sus estudios se convertirá en su nuevo estilo de vida. Es una decisión acertada poner como requisito la aprobación de 640 horas en prácticas pre profesionales en cualquier institución, porque se convierte en un recurso de aplicación de conocimientos adquiridos en la universidad. En la presente investigación, se busca resaltar la importancia del desarrollo de las prácticas, así como la identificación de las etapas que intervienen a lo largo de la formación académica; donde se identificaron: la observación, ayudantía docente y la práctica real. La primera etapa tiene como objetivo observar las metodologías de enseñanza del idioma inglés usadas en el sistema educativo, la segunda etapa consiste en evaluar a los objetivos y sujetos de estudio en el aprendizaje del idioma inglés y en la última etapa se diseña y aplica adaptaciones curriculares que promueven la inclusión, interculturalidad, equidad de género y respeto al medio ambiente. La metodología aplicada es netamente cualitativa porque se analizó la información de las actividades para cada una de las etapas llevando a cabo la aplicación de más de 6 instrumentos de recolección de datos y evaluación.
\end{abstract}

Palabras claves: experiencia, observación, ayudantía docente, práctica real

\footnotetext{
1 Docente de la Universidad Técnica de Ambato. Ecuador

2 Docente de la Universidad Técnica de Ambato. Ecuador

3 Docente de la Universidad Técnica de Ambato. Ecuador
} 


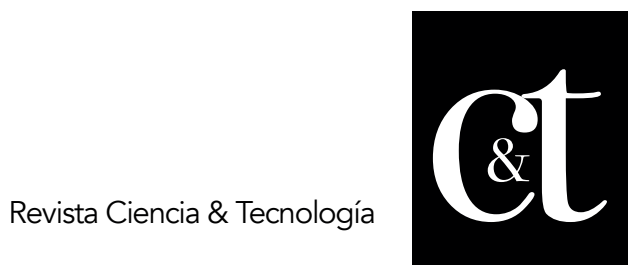

No. 17, 31 de enero de 2018

ISSN impreso: 1390 - 6321

\begin{abstract}
In today's world, most institutions request teachers with experience in teaching, and it is precisely the pre-professional practices that provide students with the required experience, because they get involved in the real world, where at the end of their studies it will become their new life style. It is a wise decision to place as a requirement the approval of 640 hours in pre-professional practices in any institution, because it becomes a resource for application of knowledge acquired in the university. In the present investigation, we want to highlight the importance of the development of the practices, as well as the identification of the stages that intervene throughout the academic formation; where they were identified: observation, teaching assistantship and real practice. The first stage aims to observe the teaching methodologies of the English language used in the educational system, the second stage consists in evaluating the objectives and subjects of study in the learning of the English language and in the last stage, curricular adaptations are designed and applied that promote inclusion, interculturality, gender equality and respect for the environment. The methodology applied is purely qualitative because the information on the activities for each of the stages was analyzed, carrying out the application of more than 6 instruments for data collection and evaluation.
\end{abstract}

Keywords: experience, observation, teaching assistantship, real practice

\title{
Introducción
}

Las prácticas pre profesionales son una parte muy importante en la formación de los estudiantes de educación superior, el Consejo de Educación Superior (2013) en el Art. 88 indica que: "las prácticas pre profesionales o pasantías son parte fundamental del currículo y serán registradas en el portafolio académico". Estas prácticas deberán ser de investigación-acción y se realizarán en el entorno institucional, empresarial o comunitario, público o privado, adecuado para el fortalecimiento del aprendizaje. Además, para las prácticas pre-profesionales se asignarán 640 horas que podrán ser distribuidas a lo largo de la carrera, dependiendo del nivel formativo y normativa existente.

Se constituyen como el principal recurso para que, el estudiante pueda desenvolverse en el mundo real que al culminar sus estudios deberá enfrentar. Para ello, debe estar preparado y aplicar todos los conocimientos que ha obtenido a lo largo de su ciclo académico, llevando un control de las principales actividades que desarrolla y los lineamientos que su carrera exige para aprobarla dentro de su malla curricular.

Es importante notar en este aspecto que las actividades que desarrolla la unidad de investigación son distintas para niveles de primero a tercer semestre; así como, el apoyo del docente para las prácticas profesionalizantes de cuarto a sexto semestre y la práctica real de séptimo semestre. Sin embargo, el objetivo sigue siendo el mismo, apoyar a los estudiantes para que se involucren en el mundo real y retengan conocimientos que luego podrán aplicar de manera más fácil. 


\section{Conceptualización}

De acuerdo a Coleman (1989) las prácticas son una experiencia laboral supervisada, de corta duración, ofrecida como parte del currículum y desarrollada durante la secuencia académica. Esto permite al estudiante desarrollar nuevas habilidades, así como aprender a desenvolverse en una cultura organizacional distinta. "Las prácticas preprofesionales constituyen un componente esencial de la formación de los estudiantes de educación superior, quienes con ellas inician su inserción en el mundo laboral por un período determinado, relacionando la práctica con la teoría" (Vega \& Arakaki, 2011, p. 78).

Por otro lado, bajo la perspectiva de León \& Mayta (2011) "es el conjunto de actividades realizadas por el practicante en forma temporal en una empresa u organización, poniendo especial énfasis en el proceso de aprendizaje y entrenamiento profesional" (p. 28) Asimismo, el Consejo de Educación Superior (2013) establece que las prácticas pre profesionales: "son actividades de aprendizaje orientadas a la aplicación de conocimientos y al desarrollo de destrezas y habilidades específicas que un estudiante debe adquirir para un adecuado desempeño en su futura profesión" ( $p$. 29).

La CES en el artículo 93, explica que las Instituciones de Educación Superior tienen la tarea de diseñar, organizar y evaluar las prácticas que desarrollan los estudiantes por cada carrera; razón por la cual, se debe llevar a cabo la implementación de programas y proyectos de vinculación, con la participación de sectores productivos, sociales y culturales tomando en cuenta las siguientes normas:

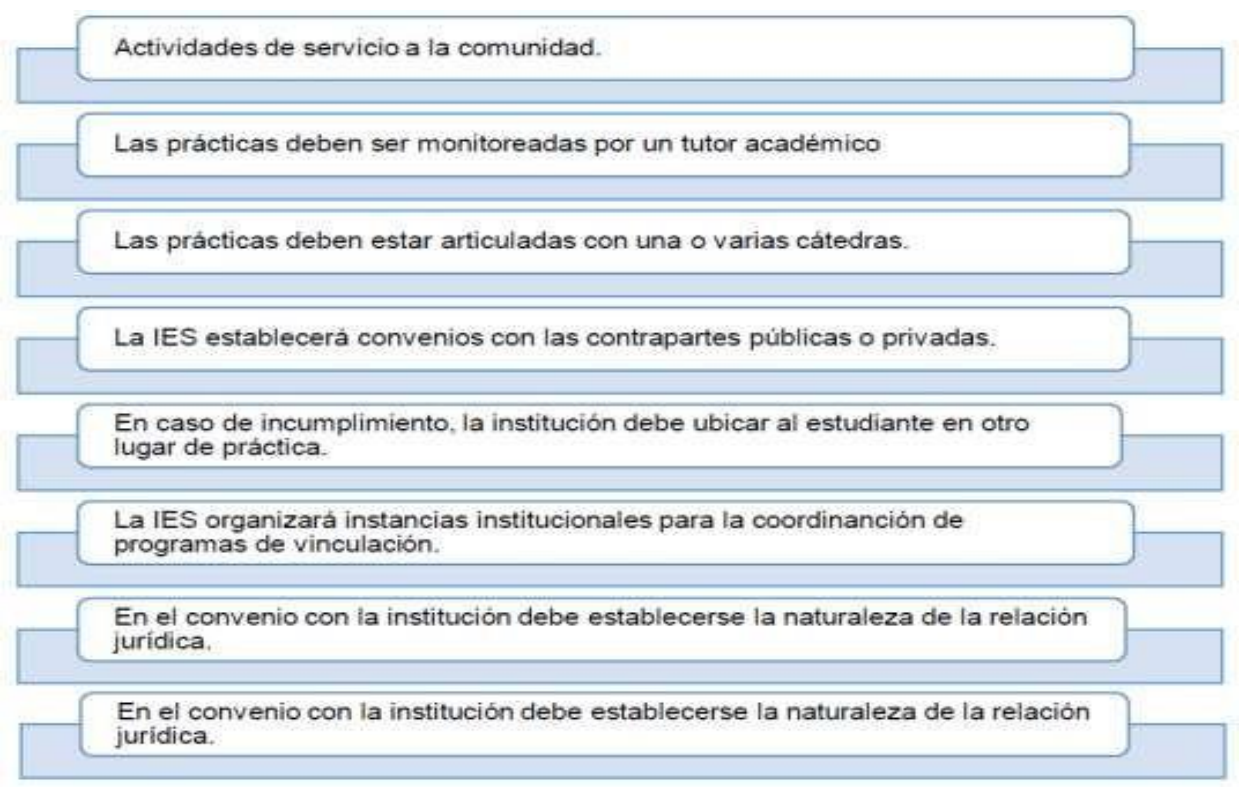

Gráfico 1. Reglamento de Régimen Académico.

Fuente: Consejo de Educación Superior (2013) "Reglamento de Régimen Académico" (p. 29)

El Art. 93 de Reglamento Académico del CES indica que: "se tomará en cuenta la 86 


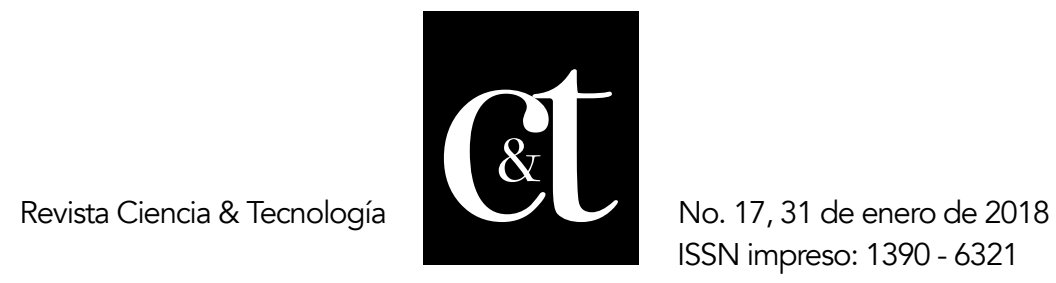

planificación y ejecución de las prácticas pre profesionales para la evaluación de carreras, considerando el cumplimiento de la presente normativa".

En la Universidad Técnica de Ambato, dentro de la Carrera de Pedagogía de los Idiomas Extranjeros, el proyecto de prácticas pre profesionales nace de la necesidad de que, tanto; los estudiantes como las instituciones educativas en convenio, puedan aprender y aplicar procesos metodológicos que aporten al desarrollo educativo. Las actividades de prácticas pre profesionales tienen el fiel propósito de observar el contexto, los sujetos y metodologías de enseñanza del idioma inglés en el sistema educativo.

La profesionalización en la formación docente es una meta impuesta a las universidades ecuatorianas (Rodríguez Morales, Milanés Gómez \& Ávila Portuondo, 2016).

\section{Desarrollo}

Unidad de Observación

Primer Semestre

Objetivo General: Identifica los propósitos de la profesión dentro de un marco políticoeducativo. 
Tabla 1. Actividades para los niveles educativos de nuestro sistema ecuatoriano: inicial, básico, bachillerato y superior (según la naturaleza de la carrera)

\begin{tabular}{|c|c|c|}
\hline $\begin{array}{l}\text { ASPECTOS DE LA } \\
\text { OBSERVACIÓN }\end{array}$ & ACTIVIDADES & $\begin{array}{l}\text { INSTRUMENTOS DE } \\
\text { RECOLECCIÓN DE } \\
\text { DATOS }\end{array}$ \\
\hline $\begin{array}{l}\text { Estructura } \\
\text { Organizacional }\end{array}$ & $\begin{array}{l}\text { Observación y análisis de la } \\
\text { estructura organizacional de } \\
\text { la institución Educativa } \\
\text { - Areas con las que cuenta. } \\
\text { - Funciones que cumple cada } \\
\text { uno de los ámbitos. } \\
\text { - Actividades que cumple cada } \\
\text { funcionario en su respectivo } \\
\text { cargo. } \\
\text { - Administración jerárquica de la } \\
\text { institución. } \\
\text {-Ámbitos con los que cuenta la } \\
\text { institución educativa para el área } \\
\text { de inglés: } \\
\text { Funciones y actividades que se } \\
\text { cumple en cada uno de los } \\
\text { ambitos que dispone la } \\
\text { institución educativa. }\end{array}$ & $\begin{array}{c}\text { Ficha de } \\
\text { Observación } 1 \\
\text { Diario de Campo }\end{array}$ \\
\hline Infraestructura & $\begin{array}{l}\text { Observación y análisis de la } \\
\text { infraestructura y equipos con } \\
\text { los que cuenta la institución } \\
\text { educativa para la enseñanza } \\
\text { del idioma inglés. } \\
\text { - Número de aulas, sillas, } \\
\text { grabadoras, tvs, laboratorios, } \\
\text { coliseo y auditorio cuenta la } \\
\text { institución para la enseñanza del } \\
\text { idioma inglés. } \\
\text {-Tecnologia con la que cuenta } \\
\text { la institución para el aprendizaje } \\
\text { del idioma inglés. }\end{array}$ & $\begin{array}{c}\text { Ficha de } \\
\text { observación } 2 \\
\text { Diario de Campo }\end{array}$ \\
\hline
\end{tabular}




\begin{tabular}{|c|c|c|}
\hline $\begin{array}{l}\text { Curriculo Nacional } \\
\text { y Micro } \\
\text { Planificación }\end{array}$ & $\begin{array}{l}\text { Observación y análisis del } \\
\text { curriculo Nacional y Micro } \\
\text { Planificación curricular del } \\
\text { idioma inglés. } \\
\text { Análisis del Currículo Nacional } \\
\text { del Idioma Inglés y la } \\
\text { planificación micro curricular. }\end{array}$ & $\begin{array}{c}\text { Ficha de } \\
\text { Observación } 3 \\
\text { Diario de Campo }\end{array}$ \\
\hline $\begin{array}{l}\text { Recursos } \\
\text { Didácticos }\end{array}$ & $\begin{array}{l}\text { Observación y análisis de los } \\
\text { recursos didácticos que se } \\
\text { utilizan para la enseñanza del } \\
\text { idioma inglés } \\
\text { - Que material didáctico se utiliza } \\
\text { para el aprendizaje del idioma } \\
\text { inglés, (texto, material audio- } \\
\text { visual, otros en caso de existirlo). }\end{array}$ & $\begin{array}{c}\text { Ficha de } \\
\text { Observación } 4 \\
\text { Diario de Campo }\end{array}$ \\
\hline $\begin{array}{l}\text { Sujetos que } \\
\text { intervienen en el } \\
\text { proceso EA }\end{array}$ & $\begin{array}{l}\text { Observación y análisis de los } \\
\text { sujetos que intervienen en el } \\
\text { proceso de aprendizaje. } \\
\text { (Nivel Inicial, Básico, } \\
\text { Bachillerato, Superior). } \\
\text { Funciones que cumple el } \\
\text { docente: Tales como: } \\
\text { asistencia a reunión de } \\
\text { area, la enseñanza del } \\
\text { idioma, planificación, } \\
\text { preparación de material } \\
\text { didáctico, adaptación } \\
\text { curricular y evaluación. } \\
\text { Las edades de los } \\
\text { estudiantes. } \\
\text { Existe participación activa } \\
\text { de los estudiantes en el } \\
\text { aprendizaje. } \\
\text { Utilizan el idioma dentro del } \\
\text { aula de clase y fuera de ella. } \\
\text { La disciplina de los } \\
\text { estudiantes. } \\
\text { El clima social escolar } \\
\text { dentro del aula. } \\
\text { Presentan deberes. } \\
\text { Ponen atención al docente. }\end{array}$ & $\begin{array}{c}\text { Ficha de } \\
\text { Observación } 5 \\
\text { Diario de Campo }\end{array}$ \\
\hline
\end{tabular}

Elaborado por: Autores 
Segundo Semestre

Objetivo General: Estas actividades están caracterizadas porque los sujetos mantienen una interacción con las comunidades de aprendizaje.

\section{Tabla 2. Actividades de prácticas pre profesionales de segundo semestre}

\begin{tabular}{|c|c|c|}
\hline $\begin{array}{c}\text { ASPECTOS DE } \\
\text { LA } \\
\text { OBSERVACIÓN }\end{array}$ & ACTIVIDADES & $\begin{array}{l}\text { INSTRUMENTOS DE } \\
\text { EVALUACIÓN }\end{array}$ \\
\hline $\begin{array}{l}\text { Interacción } \\
\text { Estudiante- } \\
\text { Estudiante }\end{array}$ & $\begin{array}{l}\text { Actividad } 1 \\
\text { Observación y analisis de la } \\
\text { interacción existente entre } \\
\text { estudiante-estudiante. } \\
\text { Interacciones a nivel de } \\
\text { enseñanza-aprendizaje. } \\
\text { Trabajo cooperativo (trabajo en } \\
\text { pares). } \\
\text { Conflictos y resolución de } \\
\text { conflictos. }\end{array}$ & $\begin{array}{c}\text { Ficha de } \\
\text { Observación } 1 \\
\text { Diario de Campo }\end{array}$ \\
\hline $\begin{array}{l}\text { Interacción } \\
\text { Estudiante- } \\
\text { Docente }\end{array}$ & $\begin{array}{l}\text { Actividad } 2 \\
\text { Observación y analisis de la } \\
\text { interacción estudiante-profesor. } \\
\quad \text { Nivel de confianza. } \\
\text { - Niveles de ansiedad. } \\
\quad \text { Nivel de respeto. } \\
\text { Preguntas al docente. }\end{array}$ & $\begin{array}{c}\text { Ficha de } \\
\text { Observación } 2 \\
\text { Diario de Campo }\end{array}$ \\
\hline $\begin{array}{l}\text { Interacción } \\
\text { Docente- } \\
\text { Estudiante }\end{array}$ & $\begin{array}{l}\text { Actividad } 3 \\
\text { Observación y analisis de la } \\
\text { interacción profesor-estudiante. } \\
\text { Instrucción de actividades } \\
\text { (claras, precisas, concisas, } \\
\text { ambiguas). } \\
\text { - Apertura. } \\
\text { Manejo de disciplina. } \\
\text { Monitoreo de actividades. }\end{array}$ & $\begin{array}{c}\text { Ficha de } \\
\text { Observación } 3 \\
\text { Diario de Campo }\end{array}$ \\
\hline $\begin{array}{l}\text { Interacción } \\
\text { Docente- } \\
\text { Docente }\end{array}$ & $\begin{array}{l}\text { Actividad } 4 \\
\text { Observación y análisis de la } \\
\text { interacción profesor-profesor. } \\
\text { Relación comunicación entre } \\
\text { docentes. } \\
\text { Relación entre docentes y } \\
\text { autoridades. } \\
\text { Ambiente laboral. } \\
\text { Formalidad e informalidad en } \\
\text { reuniones (registro). }\end{array}$ & $\begin{array}{c}\text { Ficha de entrevista } \\
\text { Informe de } \\
\text { entrevista }\end{array}$ \\
\hline $\begin{array}{l}\text { Interacción } \\
\text { Docente- } \\
\text { Comunidad }\end{array}$ & $\begin{array}{l}\text { Actividad 5 } \\
\text { Observación y analisis de la } \\
\text { interacción estudiante profesor- } \\
\text { comunidad. } \\
\text { - Actividades extracurriculares. } \\
\text { Interacciones sociales en el } \\
\text { mundo real. } \\
\text { Interacciones sociales en } \\
\text { linea. }\end{array}$ & $\begin{array}{c}\text { Ficha de } \\
\text { Observación } 5 \\
\text { Diario de Campo }\end{array}$ \\
\hline
\end{tabular}

Elaborado por: Autores 
Tercer Semestre

Objetivo general: Estas actividades se caracterizan porque reconocen las teorías y los modelos pedagógicos que se aplican en el proceso educativo.

Tabla 3. Actividades de prácticas pre profesionales de tercer semestre

\begin{tabular}{|c|c|c|}
\hline $\begin{array}{c}\text { ASPECTOS DE } \\
\text { LA } \\
\text { OBSERVACIÓN }\end{array}$ & ACTIVIDADES & $\begin{array}{l}\text { INSTRUMENTOS DE } \\
\text { EVALUACIÓN }\end{array}$ \\
\hline $\begin{array}{l}\text { Modelos } \\
\text { Pedagógicos }\end{array}$ & $\begin{array}{l}\text { Actividad } 1 \\
\text { - Observar e identificar los modelos } \\
\text { pedagógicos aplicados en la } \\
\text { enseñanza del idioma inglés. }\end{array}$ & $\begin{array}{c}\text { Ficha de } \\
\text { Observación } \\
\text { Diario de Campo }\end{array}$ \\
\hline $\begin{array}{l}\text { Modelos } \\
\text { Pedagógicos }\end{array}$ & $\begin{array}{l}\text { Actividad } 2 \\
\text { - Análisis de los modelos } \\
\text { pedagógicos utilizados por el } \\
\text { docente en el proceso de } \\
\text { enseñanza del idioma inglés. }\end{array}$ & $\begin{array}{c}\text { Ficha de } \\
\text { Observación } \\
\text { Diario de Campo }\end{array}$ \\
\hline $\begin{array}{l}\text { Modelos } \\
\text { Pedagógicos }\end{array}$ & $\begin{array}{l}\text { Actividad } 3 \\
\text { - Descripción de los modelos } \\
\text { pedagógicos utilizados por el } \\
\text { docente en el proceso de } \\
\text { enseñanza del idioma inglés. }\end{array}$ & $\begin{array}{c}\text { Ficha de } \\
\text { Observación } \\
\text { Diario de Campo }\end{array}$ \\
\hline $\begin{array}{l}\text { Modelos } \\
\text { Pedagógicos }\end{array}$ & $\begin{array}{l}\text { Actividad } 4 \\
\text { Comparación de los modelos } \\
\text { pedagógicos utilizados para la } \\
\text { enseñanza del idioma inglés. }\end{array}$ & $\begin{array}{c}\text { Ficha de } \\
\text { Observación } \\
\text { Diario de Campo }\end{array}$ \\
\hline
\end{tabular}

Elaborado por: Autores

\section{Ayudantía Docente}

Cuarto - Séptimo Semestre

Objetivo general: Describe la aplicación de los modelos donde el sujeto puede aprender en ambientes educativos reales. 
Tabla 4. Actividades de prácticas pre profesionales de la unidad profesionalizante de los semestres: cuarto - séptimo

\begin{tabular}{|c|c|c|}
\hline $\begin{array}{l}\text { ASPECTOS DEL } \\
\text { ACOMPAÑAMIENTO }\end{array}$ & ACTIVIDADES & $\begin{array}{l}\text { INSTRUMENTOS } \\
\text { DE EVALUACIÓN }\end{array}$ \\
\hline $\begin{array}{l}\text { Asistencia } \\
\text { Pedagógica }\end{array}$ & $\begin{array}{l}\text { Actividad } 1 \\
\text { Asistencia en la ejecución de } \\
\text { actividades pedagógicas. } \\
\text { Asistencia en la elaboración de } \\
\text { material didáctico bajo la } \\
\text { supervisión del tutor. } \\
\text { Corrección de trabajos, tareas y } \\
\text { evaluaciones objetivas. } \\
\text { Monitoreo de trabajos grupales. } \\
\text { Asistencia con notificaciones } \\
\text { básicas del docente. } \\
\text { Supervisión de la disciplina de los } \\
\text { estudiantes. }\end{array}$ & Portafolio \\
\hline $\begin{array}{l}\text { Estrategias } \\
\text { Comunicativas }\end{array}$ & $\begin{array}{l}\text { Actividad } 2 \\
\text { Descripción de estrategias } \\
\text { comunicativas. }\end{array}$ & Ficha descriptiva \\
\hline Lesson Study & $\begin{array}{l}\text { Actividad } 3 \\
\text { Planificación y ejecución de } \\
\text { actividades de calentamiento } \\
\text { (warm up activities). }\end{array}$ & Planificación \\
\hline
\end{tabular}

Elaborado por: Autores

Quinto Semestre

Objetivo general: Reflexiona sobre los modelos didácticos (socrático, activo situado, aprendizaje para el dominio, comunicativo interactivo, contextual ecológico, colaborativo) que se utilizan en el contexto educativo. 


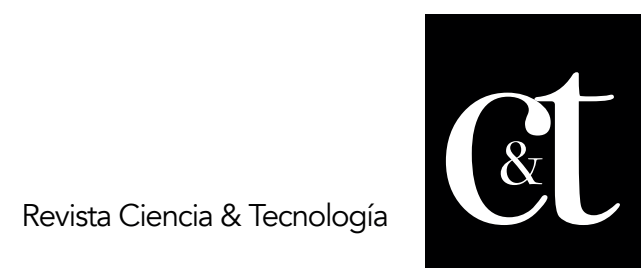

No. 17, 31 de enero de 2018

ISSN impreso: 1390 - 6321

Tabla 5. Actividades de prácticas pre profesionales de la unidad profesionalizante de quinto semestre

\begin{tabular}{|c|c|c|}
\hline $\begin{array}{c}\text { ASPECTOS DEL } \\
\text { ACOMPAÑAMIENTO }\end{array}$ & ACTIVIDADES & $\begin{array}{l}\text { INSTRUMENTOS } \\
\text { DE EVALUACIÓN }\end{array}$ \\
\hline \multirow[t]{2}{*}{$\begin{array}{l}\text { Asistencia } \\
\text { Pedagógica }\end{array}$} & $\begin{array}{l}\text { Actividad } 1 \\
\text { Asistencia en la ejecución de } \\
\text { actividades pedagógicas. } \\
\text { Elaboración de material } \\
\text { didáctico. } \\
\text { Corrección de trabajos, tareas y } \\
\text { evaluaciones objetivas. } \\
\text { Monitoreo de trabajos grupales. }\end{array}$ & Portfolio \\
\hline & $\begin{array}{l}\text { Asistencia con notificaciones } \\
\text { básicas del docente. } \\
\text { Supervisión de la disciplina de los } \\
\text { estudiantes. } \\
\text { Asistencia en la planificación } \\
\text { anual, unidad didáctica. }\end{array}$ & \\
\hline Manejo de clase & $\begin{array}{l}\text { Actividad } 2 \\
\text { Reflexiona y analizar aspectos } \\
\text { del manejo de clase. }\end{array}$ & $\begin{array}{l}\text { Registro } \\
\text { anecdótico }\end{array}$ \\
\hline $\begin{array}{l}\text { Actividades } \\
\text { docentes }\end{array}$ & $\begin{array}{l}\text { Actividad } 3 \\
\text { Reflexiona acerca de las } \\
\text { actividades docentes como } \\
\text { entrevistas con los padres de } \\
\text { familia. }\end{array}$ & $\begin{array}{c}\text { Registro } \\
\text { descriptivo }\end{array}$ \\
\hline Lesson study & $\begin{array}{l}\text { Actividad } 4 \\
\text { Planificación y ejecución de } \\
\text { actividades de clase y de cierre } \\
\text { de clase (wrap up). }\end{array}$ & $\begin{array}{c}\text { Planificación de } \\
\text { clase }\end{array}$ \\
\hline
\end{tabular}

Elaborado por: Autores

Sexto Semestre

Objetivo general: Evalúa la aplicación de recursos (incluido los tecnológicos) y estrategias educativas desde el contexto educativo real. 
Tabla 6. Actividades de prácticas pre profesionales de la unidad profesionalizante de sexto semestre

\begin{tabular}{|c|c|c|}
\hline $\begin{array}{c}\text { ASPECTOS DEL } \\
\text { ACOMPAÑAMIENTO }\end{array}$ & ACTIVIDADES & $\begin{array}{l}\text { INSTRUMENTOS } \\
\text { DE EVALUACIÓN }\end{array}$ \\
\hline $\begin{array}{l}\text { Asistencia } \\
\text { Pedagógica }\end{array}$ & $\begin{array}{l}\text { Actividad } 1 \\
\text { Elaboración de instrumentos de } \\
\text { evaluación. } \\
\text { Monitoreo de trabajos grupales e } \\
\text { individuales. } \\
\text { Asistencia con notificaciones del } \\
\text { docente. } \\
\text { Supervisión de la disciplina de los } \\
\text { estudiantes. }\end{array}$ & $\begin{array}{l}\text { Planificación de } \\
\text { clase } \\
\text { Portafolio }\end{array}$ \\
\hline Material Didáctico & $\begin{array}{l}\text { Actividad } 2 \\
\text { Elaboración de material didáctico } \\
\text { con o sin el uso de la tecnología. }\end{array}$ & Lista de cotejo \\
\hline $\begin{array}{l}\text { Clases con base a } \\
\text { Lesson Study }\end{array}$ & $\begin{array}{l}\text { Actividad } 3 \\
\text { Preparar y dictar la clase de } \\
\text { acuerdo a la planificación del } \\
\text { tutor institucional. }\end{array}$ & $\begin{array}{c}\text { Rúbrica de } \\
\text { evaluación de clase }\end{array}$ \\
\hline
\end{tabular}

Elaborado por: Autores

Práctica Real Séptimo Semestre

Objetivo General: El objetivo en este semestre es diseñar y aplicar adaptaciones curriculares que promueven la inclusión, interculturalidad, equidad de género y respeto al medio ambiente.

Tabla 7. Actividades de práctica pre profesionales de la unidad profesionalizante de séptimo semestre

\begin{tabular}{|c|c|c|c|}
\hline TEMÁTICAS & ACTIVIDADES & RECURSOS & EVALUACIÓN \\
\hline $\begin{array}{l}\text { Adaptaciones } \\
\text { curriculares }\end{array}$ & $\begin{array}{l}\text { Actividad 1 } \\
\text { Asistencia en la ejecución de } \\
\text { actividades pedagógicas con la } \\
\text { aplicación de adaptaciones } \\
\text { curriculares. } \\
\text { Elaboración de material } \\
\text { didáctico. } \\
\text { Corrección de trabajos, tareas } \\
\text { y evaluaciones objetivas } \\
\text { Monitoreo de trabajos } \\
\text { grupales. } \\
\text { Asistencia con notificaciones } \\
\text { básicas del docente. } \\
\text { Supervisión de la disciplina de } \\
\text { los estudiantes. } \\
\text { Asistencia en la planificación } \\
\text { anual, unidad didáctica. }\end{array}$ & $\begin{array}{c}\text { Material } \\
\text { didáctico }\end{array}$ & $\begin{array}{l}\text { Rúbrica de } \\
\text { Evaluación } \\
\text { de clase }\end{array}$ \\
\hline
\end{tabular}

Elaborado por: Autores

\section{Metodología}

La presente investigación tiene un enfoque cualitativo, dado que, se utilizó únicamente la información acerca de las actividades que deben desarrollar los 94 


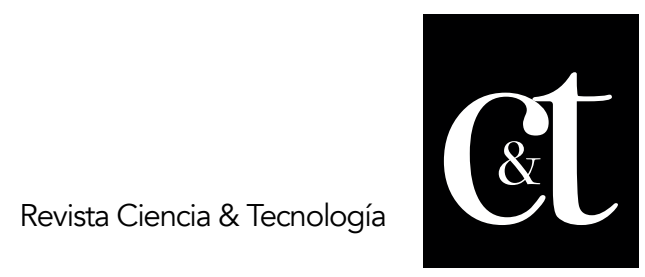

No. 17, 31 de enero de 2018

ISSN impreso: 1390 - 6321

estudiantes en su lugar de práctica pre profesional. Además, es de tipo descriptiva porque, describe cada uno de los aspectos de la observación, definiendo las principales actividades que conlleva cada proceso. Para lo cual, se utilizaron los siguientes instrumentos de evaluación en cada una de las actividades:

\section{Tabla 8. Metodología}

\begin{tabular}{|l|l|}
\hline Aspecto & Instrumento \\
\hline Estructura organizacional & Ficha de observación \\
\hline Interacción Docente-Docente & Ficha de entrevista \\
\hline Asistencia pedagógica & Portafolio \\
\hline Estrategias comunicativas & Ficha descriptiva \\
\hline Lesson Study & Planificación \\
\hline Manejo de clase & Registro anecdótico \\
\hline Actividades docentes & Registro descriptivo \\
\hline Material didáctico & Lista de cotejo \\
\hline Clases con el Lesson study & Rúbrica de evaluación \\
\hline
\end{tabular}

Fuente: Cumbe, (2018) "Proyecto de Prácticas Pre profesionales"

\section{Discusión}

Las prácticas pre profesionales constituyen aquellas actividades de aprendizaje que están encaminadas para que, el estudiante adquiera destrezas y habilidades para un desempeño idóneo en el mundo profesional. Cada uno de los semestres presentan aspectos y actividades distintas para cada proceso, en la primera parte de las actividades de las prácticas pre profesionales intervienen los niveles educativos del sistema educativo: inicial, básico, bachillerato y superior, donde se identifican los principales aspectos de la observación que no tienen que ver con el ámbito educativo, donde únicamente se realizó un análisis exploratorio en lo que se refiere a la estructura organizacional, infraestructura, currículo, recursos didácticos, interacciones entre docente y estudiante y los modelos pedagógicos. En la segunda parte, se consideró la ayudantía docente donde se evalúa los objetivos y sujetos de estudio en el aprendizaje del idioma inglés. Mientras que, en la última parte se definieron las actividades para cuarto, quinto, sexto y séptimo semestre describiendo la aplicación de modelos contextualizados, modelos didácticos, estrategias educativas y adaptaciones curriculares en ambientes educativos reales.

\section{Conclusión}

Las prácticas pre profesionales son muy importantes en la formación de los estudiantes de educación superior; dado que, esta estancia les brinda un aprendizaje práctico y real. Mediante esta información, el estudiante puede considerarse con la experiencia necesaria, que solicitan los empresarios para un puesto de trabajo.

La metodología que se utiliza es la cualitativa porque se analizó la información no numérica, únicamente se describieron las actividades que se deben desarrollar en las 3 etapas de primero a séptimo semestre; identificando los instrumentos de 95 


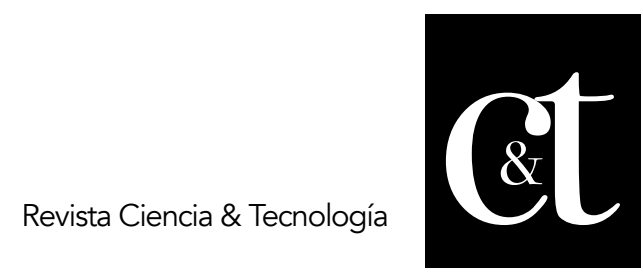

No. 17, 31 de enero de 2018

ISSN impreso: 1390 - 6321

recolección de información que fueron importantes para cada uno de los aspectos.

La investigación consta de 3 etapas: la observación donde se recopila información de manera exploratoria acerca de las actividades previas que deben desarrollarse antes de ubicarles a los estudiantes en las instituciones educativas; la ayudantía docente donde se fijan los modelos pedagógicos y las estrategias educativas; y la última parte donde ya se promueve la práctica real de las pasantías promoviendo la inclusión, interculturalidad, equidad de género y respeto de medio ambiente.

\section{Referencias Bibliográficas}

Coleman, J. (1989). The role of the practicum in library schools. Journal of Education for Library and Information Science, vol. 30, no.1. p. 19-27.

Consejo de Educación Superior CES (2013). Reglamento de Régimen Académico. Quito, Pichincha, Ecuador.

Cumbe, D. (agosto de 2018). Proyecto de prácticas pre profesionales rediseño 2016. Ambato, Tungurahua, Ecuador.

León, W., \& Mayta, R. (28 de junio de 2011). Diagnóstico de las prácticas pre profesionales: caso Facultad de Ingeniería Industrial de la UNMSM. Lima, Perú.

Rodríguez Morales, A., Milanés Gómez, R., \& Ávila Portuondo, A. M. (2016). La profesionalización una fuente para semilleros de investigación. Revista Conrado, 12(55), 83-89.

Vega, A., \& Arakaki, M. (25 de enero de 2011). Las prácticas preprofesionales en la formación en Ciencias de la Información: el caso de la Pontificia Universidad Católica del Perú (PUCP). Lima, Perú. 